\title{
Chiral response of spin-spiral states as the origin of chiral transport fingerprints of spin textures
}

\author{
Jonathan Kipp $\odot,{ }^{1,2}$ Fabian R. Lux, ${ }^{3}$ and Yuriy Mokrousov ${ }^{1,3}$ \\ ${ }^{1}$ Peter Grünberg Institut and Institute for Advanced Simulation, Forschungszentrum Jülich and JARA, 52425 Jülich, Germany \\ ${ }^{2}$ Department of Physics, RWTH Aachen University, 52056 Aachen, Germany \\ ${ }^{3}$ Institute of Physics, Johannes Gutenberg University Mainz, 55099 Mainz, Germany
}

(Received 30 September 2021; accepted 11 November 2021; published 3 December 2021; corrected 7 January 2022)

\begin{abstract}
The transport properties of nontrivial spin textures are coming under closer scrutiny as the amount of experimental data and theoretical simulations is increasing. To extend the commonly accepted yet simplifying and approximate picture of transport effects taking place in systems with spatially varying magnetization, it is important to understand the transport properties of building blocks for spin textures- the homochiral spin-spiral states. In this work, by referring to phenomenological symmetry arguments based on the gradient expansion, and explicit calculations within the Kubo framework, we study the transport properties of various types of spin-spirals in a two-dimensional model with strong spin-orbit interaction. In particular, we focus on the contributions to the magnetoconductivity, the planar Hall effect, and the anomalous Hall effect, which are sensitive to the sense of chirality of the spiral states. We analyze the emergence, symmetry, and microscopic properties of the resulting chiral magnetoconductivity, chiral planar Hall effect, and chiral Hall effect in terms of spin-spiral propagation direction, cone angle, spiral pitch, and disorder strength. Our findings suggest that the presence of spin-spiral states in magnets can be readily detected in various types of magnetotransport setups. Moreover, the sizable magnitude of chiral contributions to the conductivity of skyrmions estimated from homochiral spirals implies that chiral, as opposed to topological, magnetotransport can play a prominent role for the detection of nontrivial spin textures.
\end{abstract}

DOI: 10.1103/PhysRevResearch.3.043155

\section{INTRODUCTION}

The electron dynamics taking place in diverse magnetization textures is one of the most intensively pursued areas of solid-state physics, at the crossroads between topology, transport, and physics of nonuniform media. In particular, the transport manifestations of electron dynamics in spin textures exposed to external electric fields have come to occupy a very important role in modern skyrmionics and spintronics due to numerous implications for practical implementations of skyrmion-based design philosophy. Since the early days of the field, a commonly accepted approach for treating the transverse transport of complex spin-textures in two dimensions, such as skyrmions, has relied heavily on the presence of the so-called emergent field, coupling spatial gradients $\partial_{x} \hat{\mathbf{n}}$ and $\partial_{y} \hat{\mathbf{n}}$. Since the seminal paper of Bruno and co-workers [1], the validity of the ansatz for the transverse Hall conductivity as an object directly proportional to the emergent field has been tested and validated in many cases, from effective models [2-4], microscopic calculations $[5,6]$, and direct comparison to experiments $[7,8]$, while "topological" contributions have also been reported

Published by the American Physical Society under the terms of the Creative Commons Attribution 4.0 International license. Further distribution of this work must maintain attribution to the author(s) and the published article's title, journal citation, and DOI. for magnetoconductivity and the planar Hall effect [9-13]. However, in recent years, evidence has started to accumulate that the simple picture of the emergent field and the corresponding topological Hall effect often do not suffice to explain results from experimental transport measurements. The community has become more aware of the fact that the emergent field picture has to be radically extended [14,15,17], particularly in strongly spin-orbit coupled systems. On the one hand, recent works have shown that in spin-orbit coupled systems, the second order in magnetization gradient terms, which go beyond the conventional topological-like signal, can contribute significantly to the orbital magnetism and Hall effect exhibited by the textures $[16,17]$. On the other hand, it has been demonstrated that an effect that is much more pronounced in strongly spin-orbit coupled systems, since it is linear in the gradients of $\hat{\mathbf{n}}$, can emerge in the context of Hall currents and orbital magnetism in generic spin textures [18]. The corresponding chiral Hall effect (CHE) was shown to be directly sensitive to the sense of local spin chirality and, in contrast to the topological Hall effect, to fine details of spin distribution [18,19], which potentially makes it a powerful tool for tracking texture dynamics with magnetotransport means. Recently, it was also demonstrated from model considerations and first-principles calculations that the CHE can be prominent in canted spin-orbit coupled ferromagnets and antiferromagnets [20]. For textures, a signal consistent with such chiral contributions has been reported experimentally for the anomalous Hall effect (AHE) [21], the planar Hall effect (PHE) [9], and magnetoconductivity 
(MC), although additional analysis is necessary to unambiguously pin down the exact microscopic origin of the observed signal.

In the context of chiral contributions to the magnetotransport of complex spin textures, understanding chiral transport properties of elemental one-dimensional spin-spiral states presents an important milestone and a pivotal starting point. Spin-spirals have emerged as the ground state of various transition-metal compounds in bulk [7], at surfaces [22], and in one-dimensional systems [23] as a result of complex exchange interactions. Further, spin-spirals serve as natural building blocks for more complex spin structures such as multi- $q$ states [24], domain walls [25], and skyrmions [7,26], while their treatment often provides a key to modeling fluctuating magnets [27,28]. Proper theoretical understanding of symmetry and microscopic mechanisms behind the transport properties of spin-spiral states thus provides a necessary foundation for building a coherent picture of transport phenomena in diverse spin textures. A distinct transport signal of spiral states has been observed in experiments lately [21], and a recent theoretical work based on an effective Rashba model predicted the emergence of the chiral Hall effect for specific types of spin-spiral states [29]. At this point, a comprehensive picture of magnetotransport of spin-spirals grounded in a realistic electronic structure on a specific lattice has emerged as a necessary next step. Technically, computing transport properties of extended spin-spiral states is very challenging. The spin-spiral order in combination with spin-orbit interaction is inconsistent with the generalized Bloch theorem [30,31], which necessitates the use of very large unit cells containing thousands of atoms. The formidable computational effort explains the noticeable lack of expansive studies in this direction (see, e.g., [25,32]).

In our work, we study in detail the magnetotransport properties of spin-spiral states in two-dimensional magnets, and we make predictions concerning the impact of these properties on chiral transport fingerprints of large skyrmions. We refer to an effective tight-binding model of electrons on a honeycomb lattice for explicit calculations of transport properties from the electronic structure of spin-spiral states in the Kubo linear-response formalism. Inspecting components of the conductivity tensor (anti)symmetrized with respect to chirality, we uncover chiral contributions to anisotropic magnetoconductivity (denoted below as MC), the planar Hall effect, and the anomalous Hall effect. Specifically, we find perfect agreement between numerical results from the Kubo formalism and symmetry-based predictions from the gradient expansion for the existence of chiral contributions as a function of the spin-spiral propagation direction and the type of spiral. We address intrinsic and extrinsic origins of the considered effects, uncovering a nontrivial competition of disorder and Berry phase effects on the chiral Hall effect exhibited by spinspirals and skyrmions. The strong and extremely nontrivial response of the chiral transport to the pitch of the spirals found here suggests that chiral magnetotransport serves as a unique marker of the fine details of the spin distribution in spin textures. Finally, we show how by extracting effective parameters from explicit calculations of spin-spirals, the chiral transport properties of large-scale skyrmions can be predicted.

\section{APPROACH}

\section{A. Model}

We investigate the existence and properties of chiral magnetotransport effects on a bipartite honeycomb lattice of magnetic spins. To model the electronic structure, we employ an effective two-dimensional lattice tight-binding Hamiltonian (in the $x y$-plane) which reads

$$
\begin{aligned}
H= & -t \sum_{\langle i j\rangle \alpha} c_{i \alpha}^{\dagger} c_{j \alpha}+i \alpha_{\mathrm{R}} \sum_{\langle i j\rangle \alpha \beta} \hat{\mathbf{e}}_{z} \cdot\left(\boldsymbol{\sigma} \times \mathbf{d}_{i j}\right)_{\alpha \beta} c_{i \alpha}^{\dagger} c_{j \beta} \\
& +\lambda_{\operatorname{ex}} \sum_{i \alpha \beta}\left(\hat{\mathbf{s}}_{i} \cdot \boldsymbol{\sigma}\right)_{\alpha \beta} c_{i \alpha}^{\dagger} c_{i \beta},
\end{aligned}
$$

where $c_{i \alpha}^{\dagger}\left(c_{i \alpha}\right)$ denotes the creation (annihilation) of an electron with spin $\alpha$ at site $i,\langle\cdots\rangle$ restricts the sums to nearest neighbors, the unit vector $\mathbf{d}_{i j}$ points from $j$ to $i$, and $\sigma$ stands for the vector of Pauli matrices. Besides the hopping with amplitude $t$, Eq. (1) contains the Rashba spin-orbit coupling of strength $\alpha_{\mathrm{R}}$ originating, for example, in the surface potential gradient perpendicular to the plane (i.e., along $\hat{z}$ ). The remaining term in Eq. (1) is the local exchange term, with $\lambda_{\text {ex }}$ characterizing the strength of exchange splitting, and $\hat{\mathbf{s}}_{i}$ stands for the direction of spin on site $i$. Here, we work with the following parameters of the model: $t=1.0 \mathrm{eV}, \alpha_{\mathrm{R}}=0.4 \mathrm{eV}$, and $\lambda_{\text {ex }}=1.4 \mathrm{eV}$, which corresponds to the case of a strongly spin-orbit-coupled magnet.

\section{B. Parametrization of spin spirals}

In this work, we impose magnetic spiral distribution of spins $\hat{\mathbf{s}}_{i}$ in the Hamiltonian (1), and we study the transport properties of such states. We proceed by first defining a continuous, normalized vector field $\hat{\mathbf{n}}(\mathbf{x})$ which is discretized on the lattice as

$$
\hat{\boldsymbol{s}}_{i} \equiv \hat{\mathbf{n}}\left(\mathbf{R}_{i}-\mathbf{R}_{0}\right),
$$

where $\mathbf{R}_{i}$ corresponds to the real-space position of lattice site $i$, and $\mathbf{R}_{0}$ represents a choice for the origin of the continuous coordinate system. General spin-spirals are one-dimensional, periodic patterns of spins, whose modulation is characterized by a single phase factor $\Psi(\mathbf{x})=\mathbf{q} \cdot \mathbf{x}$. Explicitly, we first construct such a spin-spiral in the $x$-direction followed by a rotation $R_{\phi_{q}}^{\mathbf{e}_{z}}$ around the $z$-axis into the actual direction of the $\mathbf{q}$-vector, as characterized by its polar coordinate $\phi_{q}$ :

$$
\hat{\mathbf{n}}=R_{\phi_{q}}^{\mathbf{e}_{z}} R_{\Psi}^{\mathbf{e}_{r}} \hat{\mathbf{n}}_{0} \text {. }
$$

Here, $\hat{\mathbf{n}}_{0}$ defines the initial orientation of $\hat{\mathbf{n}}$ for $\Psi=0$. Following the direction of $\mathbf{q}$ in real space, the pattern describes a rotational motion around the axis defined by $\mathbf{e}_{r}$. With $\hat{\mathbf{n}}_{0}=\mathbf{e}_{z}$ fixed, three different cases are considered here; see Fig. 1: the Bloch spiral $\mathbf{e}_{r}=\mathbf{e}_{x}$, the Néel spiral $\mathbf{e}_{r}=\mathbf{e}_{y}$, and a tilted conical Néel phase $\mathbf{e}_{r}=\sin \alpha \mathbf{e}_{y}+\cos \alpha \mathbf{e}_{z}$ with cone angle $0 \leqslant \alpha \leqslant \pi / 2$, interpolating between the ferromagnetic phase for $\alpha=0$ and the Néel spiral for $\alpha=\pi / 2$. All different cases are contained in the parametrization

$$
\mathbf{e}_{r}=\sin \beta \sin \alpha \mathbf{e}_{x}+\cos \beta \sin \alpha \mathbf{e}_{y}+\cos \alpha \mathbf{e}_{z},
$$

where the angle $0 \leqslant \beta \leqslant \pi / 2$ can rotate from the tilted Néel phase $(\beta=0)$ to a tilted Bloch phase $(\beta=\pi / 2)$. 


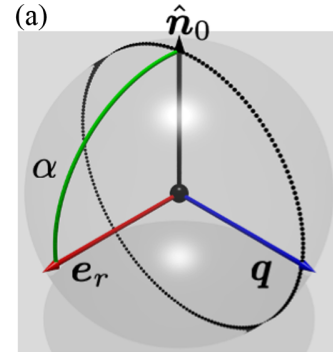

(d)

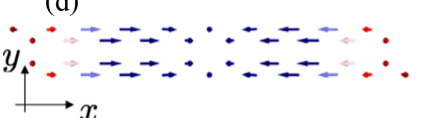

(b)

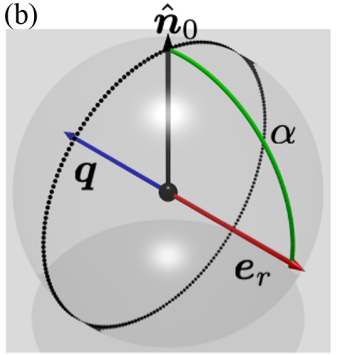

(e)

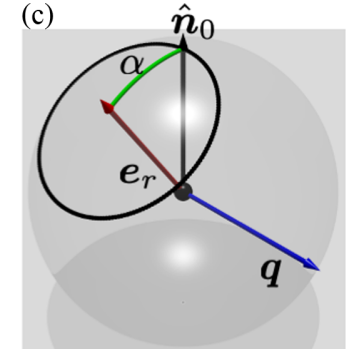

(f)

$\therefore t_{1+1}^{+\cdots}+\cdots+1+1+1+$

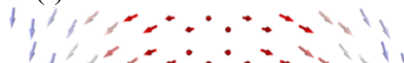

FIG. 1. Parametrization of Néel, Bloch, and cone-type spirals. Parts (a)-(c) show the parametrization of Néel (a), Bloch (b), and cone-type (c) spin-spirals on a sphere spanned by magnetic moments. The spiral propagates along a direction $\mathbf{q}$, and the magnetization rotates around an axis $\mathbf{e}_{r}$, which encloses a cone angle $\alpha$ with the initial magnetic moment $\hat{\boldsymbol{n}}_{0}$. For the Néel spiral, $\mathbf{e}_{r} \perp \mathbf{q}$, while for the Bloch spiral, $\mathbf{e}_{r} \| \mathbf{q}$. In this work, the plane of the honeycomb lattice is the $x y$-plane (axis $\hat{z}$ is perpendicular to it), which contains q. Plots (d)-(f) show the real-space distribution (as seen from the top) of corresponding magnetization textures for $\mathbf{q} \| \hat{\boldsymbol{x}}$.

The wave-vector pitch $q=\|\mathbf{q}\|$ is the main tuning knob to adjust the magnetization pattern in this work. The limiting cases are the ferromagnetic (FM) pattern for $q=0$ and the antiferromagnetic pattern for $q=\pi$. The $q=0$ limit corresponds to the regular out-of-plane (along the $z$-axis) FM case. However, depending on the orientation of the rotation axis $\mathbf{e}_{r}$ in real space, the two spins in the AFM pattern are not antiparallel, but enclose a polar angle of $2 \alpha$, twice the cone angle that the rotation axis $\mathbf{e}_{r}$ encloses with the $z$-axis. This can be understood by realizing that a phase $\pi$ between spins on neighboring atomic sites corresponds to opposite positions on the cone around the rotation axis $\mathbf{e}_{r}$, which has an opening angle of $2 \alpha$.

\section{Gradient expansion}

Within the linear-response theory, the conductivity tensor $\sigma_{\alpha \beta}$ describes the electric current response in the system, which is linear in electric field $\mathbf{E}$ as $j^{\alpha}=\sigma_{\alpha \beta} E^{\beta}$. To arrive at a way to categorize different physical effects that are encapsulated in the conductivity tensor, we start by writing an asymptotic expansion in the gradients $\partial_{i} n_{j}$ for a smooth magnetization texture, i.e.,

$$
\sigma_{\alpha \beta}[\hat{\mathbf{n}}]=\left\langle\sigma_{\alpha \beta}^{\mathrm{col}}(\hat{\mathbf{n}})+\sigma_{\alpha \beta \gamma \delta}^{\chi}(\hat{\mathbf{n}}) \partial_{\gamma} n_{\delta}+O\left(\partial^{2}\right)\right\rangle .
$$

Here, the bracket $\langle\bullet\rangle$ indicates the real-space integral $\int d \mathbf{x} / \mathcal{V}$, where $\mathcal{V}$ is the real-space volume of the system, $\mathbf{x}=(x, y)$ is the position vector, and the notation $[\hat{\mathbf{n}}]$ implies the functional dependence on the overall texture as given by the distribution of $\hat{\mathbf{n}}$ in real space. The conductivity tensor itself can be decomposed into symmetric and antisymmetric components with respect to the interchange of indices $\alpha$ and $\beta$ : $\sigma_{\alpha \beta}[\hat{\mathbf{n}}]=\sigma_{[\alpha \beta]}[\hat{\mathbf{n}}]+\sigma_{(\alpha \beta)}[\hat{\mathbf{n}}]$. Here $[\alpha \beta]$ indicates the antisymmetrization, whereas $(\alpha \beta)$ represents a symmetrization of indices. Via the Onsager reciprocity relations, $\sigma_{[\alpha \beta]}[\hat{\mathbf{n}}]=$ $-\sigma_{[\alpha \beta]}[-\hat{\mathbf{n}}]$ and $\sigma_{(\alpha \beta)}[\hat{\mathbf{n}}]=\sigma_{(\alpha \beta)}[-\hat{\mathbf{n}}]$. The symmetric tensor then describes magnetoconductivity, anisotropic magnetoconductivity, and planar Hall effects, while its antisymmetric counterpart captures the anomalous Hall effect. The superscript "col" indicates that $\sigma_{\alpha \beta}^{\mathrm{col}}(\hat{\mathbf{n}}(\mathbf{x}))$ fully describes the linear response of a collinear magnetized state. For slowly varying textures, the next-to-leading-order term $\sigma_{\alpha \beta \gamma \delta}^{\chi}(\hat{\mathbf{n}}(\mathbf{x}))$ couples to the first-order gradients of the magnetization texture and is therefore sensitive to the chirality of $\hat{\mathbf{n}}$, thereby providing the chiral part of the overall conductivity tensor. When the texture constitutes a spin-spiral with a wave vector $\mathbf{q}$, as introduced above, the corresponding chiral corrections to the conductivity are fully antisymmetric in $\mathbf{q}$.

Following [33], one can expand $\sigma^{\mathrm{col}}$ and $\sigma^{\chi}$ into powers of the magnetization vector $\hat{\mathbf{n}}$ and reduce the number of possible coupling terms using the restrictions imposed by the crystallographic point group of the nonmagnetic lattice. For example, this method has been successfully applied recently to study the chiral corrections to the spin-Hall magnetoresistance in the noncollinear magnet $\mathrm{Cu}_{2} \mathrm{OSeO}_{3}$ [34]. Below, we demonstrate the way that it can be done for the partial case of $C_{6 v}$ symmetry of the Hamiltonian as given by Eq. (1). A systematic way to perform this reduction is guided by the representation theory of $C_{6 v}$ as summarized by the character table in Table I. Accordingly, the gradient operator $\nabla=\left(\partial_{x}, \partial_{y}\right)$ generates the representations $\Gamma_{\mathbf{x}}=\Gamma_{\nabla}=E_{1}$. The magnetization $\hat{\mathbf{n}}$-as an axial vector-decomposes into $\hat{\mathbf{n}}_{\|}=\left(n_{x}, n_{y}, 0\right)$ belonging to $E_{1}$, and $\hat{\mathbf{n}}_{\perp}=\left(0,0, n_{z}\right)$ belonging to $A_{2}$, i.e., $\Gamma_{\hat{\mathbf{n}}}=A_{2}+E_{1}$. Using the Schur orthogonality relations [35,36], one can derive the decomposition of the Kronecker products $\Gamma_{i} \otimes$

TABLE I. Character table of $C_{6 v}$. Shown are the characters of each irreducible representation for each conjugacy class, alongside linear and quadratic basis functions which generate the respective representations.

\begin{tabular}{ccccccccc}
\hline \hline$C_{6 v}$ & $E$ & $2 C_{6}(\mathrm{z})$ & $2 C_{3}(\mathrm{z})$ & $C_{2}(\mathrm{z})$ & $3 \sigma_{v}$ & $3 \sigma_{d}$ & linear & quadratic \\
\hline$A_{1}$ & 1 & 1 & 1 & 1 & 1 & 1 & $z$ & $x^{2}+y^{2}, z^{2}$ \\
$A_{2}$ & 1 & 1 & 1 & 1 & -1 & -1 & $n_{z}$ & \\
$B_{1}$ & 1 & -1 & 1 & -1 & 1 & -1 & & \\
$B_{2}$ & 1 & -1 & 1 & -1 & -1 & 1 & & \\
$E_{1}$ & 2 & 1 & -1 & -2 & 0 & 0 & $(x, y)$ & $(x z, y z)$ \\
$E_{2}$ & 2 & -1 & -1 & 2 & 0 & 0 & & $\left(x^{2}-y^{2}, x y\right)$ \\
\hline \hline
\end{tabular}


TABLE II. Kronecker products in $C_{6 v}$. Shown is the reduction of all possible combinations of Kronecker products among the irreducible representations of $C_{6 v}$.

\begin{tabular}{ccccccc}
\hline \hline$\otimes$ & $A_{1}$ & $A_{2}$ & $B_{1}$ & $B_{2}$ & $E_{1}$ & $E_{2}$ \\
\hline$A_{1}$ & $A_{1}$ & $A_{2}$ & $B_{1}$ & $B_{2}$ & $E_{1}$ & $E_{2}$ \\
$A_{2}$ & $A_{2}$ & $A_{1}$ & $B_{2}$ & $B_{1}$ & $E_{1}$ & $E_{2}$ \\
$B_{1}$ & $B_{1}$ & $B_{2}$ & $A_{1}$ & $A_{2}$ & $E_{2}$ & $E_{1}$ \\
$B_{2}$ & $B_{2}$ & $B_{1}$ & $A_{2}$ & $A_{1}$ & $E_{2}$ & $E_{1}$ \\
$E_{1}$ & $E_{1}$ & $E_{1}$ & $E_{2}$ & $E_{2}$ & $A_{1}+A_{2}+E_{2}$ & $B_{1}+B_{2}+E_{1}$ \\
$E_{2}$ & $E_{2}$ & $E_{2}$ & $E_{1}$ & $E_{1}$ & $B_{1}+B_{2}+E_{1}$ & $A_{1}+A_{2}+E_{2}$ \\
\hline \hline
\end{tabular}

$\Gamma_{j}=\bigoplus_{k} \lambda_{k} \Gamma_{k}$ among any of the irreducible representations (irreps) $\Gamma_{i}$, where $\lambda_{k}$ are positive integers. Summarized in Table II, the result of this procedure can be used to study the symmetry-allowed couplings in the expansion of $\sigma_{\alpha \beta}$ : since it couples two polar vectors in the $x-y$ plane, it carries the representation $\sigma=E_{1} \otimes E_{1}=A_{1}+A_{2}+E_{2}$. Different irreps, therefore, distinguish different categories of physical effects, which are summarized in Table III and which will be introduced in the following. Due to the Onsager relations, the $A_{1}$ and $E_{2}$ contributions to $\sigma_{\alpha \beta}$ have to be even in $n_{i}$ while the $A_{2}$ term is odd. In the absence of magnetism or possible external fields, all components that do not belong to the totally symmetric representation $A_{1}$ have to vanish by Neumann's symmetry principle [37,38]. The $A_{1}$ term $\left(\sigma_{(x x)}+\sigma_{(y y)}\right) / 2$ then captures the isotropic contributions to the longitudinal conductivity. In the presence of a finite magnetization, the components of $\hat{\mathbf{n}}$ and its gradients can form a basis for the irreducible representations $A_{2}$ and $E_{2}$, thereby leading to a finite anomalous Hall effect in $\sigma_{[x y]}$ and a finite planar Hall effect in $\sigma_{(x y)}$ for example. Using representation theory, one can study what different terms appear in the gradient expansion of Eq. (5) if each of the tensors is expanded as a power series in $n_{i}$. For the collinear case, one finds that the magnetization carries the following irreducible representations:

$$
\begin{aligned}
& A_{1}: n_{\|}^{2}, n_{\perp}^{2}, \\
& A_{2}: n_{z}, \\
& E_{1}:\left(n_{x} n_{z}, n_{y} n_{z}\right), \\
& E_{2}\left(\left(n_{x}^{2}-n_{y}^{2}\right) / 2, n_{x} n_{y}\right),
\end{aligned}
$$

up to second order in $n_{i}$, where $n_{\perp}^{2}=n_{z}^{2}$ and $n_{\|}^{2}=n_{x}^{2}+n_{y}^{2}$. Here, one has to take into account that $\hat{\mathbf{n}}$ transforms as a pseudovector. At the given order in $n_{i}$, there are thus five couplings to the collinear magnetic state:

$$
\begin{aligned}
& \sigma_{A_{1}}^{\mathrm{col}}(\hat{\mathbf{n}})=\gamma_{\mathrm{LC}}+\gamma_{\mathrm{MC}}\|\hat{\mathbf{n}}\|^{2}+\gamma_{\mathrm{AMC}}\left(n_{\perp}^{2}-n_{\|}^{2}\right)+O\left(n_{i}^{4}\right), \\
& \sigma_{A_{2}}^{\mathrm{col}}(\hat{\mathbf{n}})=\gamma_{\mathrm{AHE}} n_{z}+O\left(n_{i}^{3}\right), \\
& \sigma_{E_{2}}^{\mathrm{col}}(\hat{\mathbf{n}})=\gamma_{\mathrm{PHE}}\left[\left(n_{x}^{2}-n_{y}^{2}\right) / 2, n_{x} n_{y}\right]+O\left(n_{i}^{4}\right),
\end{aligned}
$$

which we refer to as the longitudinal conductivity (LC), the magnetoconductivity (MC), the anisotropic magnetoconductivity (AMC), the anomalous Hall effect (AHE), and the planar Hall effect (PHE). The PHE is commonly understood as the off-diagonal components $\sigma_{(x y)}$ of the symmetrized conductivity tensor. Due to the peculiarity of the $C_{6 v}$ symmetry, the same coefficient controls anisotropic contributions to the longitudinal conductivity $\left(\sigma_{(x x)}-\sigma_{(y y)}\right) / 2$. In Table III, this latter term is therefore listed as the longitudinal planar Hall effect (LPHE).

Moving on to the gradient-induced effects in two dimensions, one can deduce from Tables I and II that $\Gamma_{\nabla \otimes \hat{\mathbf{n}}} \equiv$ $E_{1} \otimes\left(A_{2}+E_{2}\right)=A_{1}+A_{2}+E_{1}+E_{2}$. The relevant irrep is $A_{2}$ (due to the Onsager relations), and it occurs only once:

$$
\begin{aligned}
& A_{1}:(\nabla \times \hat{\mathbf{n}})_{z}, \\
& A_{2}: \nabla \cdot \hat{\mathbf{n}}, \\
& E_{1}:\left(\partial_{x} n_{z}, \partial_{y} n_{z}\right), \\
& E_{2}:\left(\partial_{x} n_{x}-\partial_{y} n_{y}, \partial_{x} n_{y}+\partial_{y} n_{x}\right) .
\end{aligned}
$$

We assume that partial integration can be performed under the real-space integral in Eq. (5), which renders the $A_{2}$ contribution integrate to zero. The next order in $n_{i}$ can be obtained by reducing the tensor product $\Gamma_{\nabla \otimes \hat{\mathbf{n}}} \otimes \Gamma_{\hat{\mathbf{n}}}=2 A_{1}+2 A_{2}+B_{1}+$ $B_{2}+4 E_{1}+2 E_{2}$ :

$$
\begin{aligned}
& A_{2} \otimes A_{2} \rightarrow A_{1}:(\nabla \cdot \hat{\mathbf{n}}) n_{z}, \\
& E_{1} \otimes A_{2} \rightarrow A_{1}:(\hat{\mathbf{n}} \cdot \nabla) n_{z}, \\
& E_{2} \otimes A_{2} \rightarrow E_{2}: n_{z}\left(\partial_{x} n_{x}-\partial_{y} n_{y}, \partial_{x} n_{y}+\partial_{y} n_{x}\right), \\
& E_{1} \otimes E_{1} \rightarrow E_{2}:\left(n_{x} \partial_{x} n_{z}-n_{y} \partial_{y} n_{z}, n_{x} \partial_{y} n_{z}+n_{y} \partial_{x} n_{z}\right) .
\end{aligned}
$$

\begin{tabular}{|c|c|c|c|c|}
\hline Irrep & Channel & Name & Collinear effects $O\left(\partial^{0}\right)$ & Chiral effects $O\left(\partial^{1}\right)$ \\
\hline$A_{1}$ & $\left(\sigma_{(x x)}+\sigma_{(y y)}\right) / 2$ & $\begin{array}{l}\text { isotropic } \\
\text { longitudinal }\end{array}$ & $\begin{array}{l}\text { longitudinal conductivity (LC), } \\
\text { magnetoconductivity (MC), anisotropic } \\
\text { magnetoconductivity (AMC) }\end{array}$ & chiral magnetoconductivity (CMC) \\
\hline$A_{2}$ & $\sigma_{[x y]}$ & $\begin{array}{l}\text { antisymmetric } \\
\text { transverse }\end{array}$ & anomalous Hall effect (AHE) & chiral Hall effect (CHE) \\
\hline$\left(E_{2}\right)_{1}$ & $\left(\sigma_{(x x)}-\sigma_{(y y)}\right) / 2$ & $\begin{array}{l}\text { anisotropic } \\
\text { longitudinal }\end{array}$ & longitudinal planar Hall effect (LPHE) & chiral longitudinal planar Hall effect (CLPHE) \\
\hline$\left(E_{2}\right)_{2}$ & $\sigma_{(x y)}$ & $\begin{array}{l}\text { symmetric } \\
\text { transverse }\end{array}$ & planar Hall effect (PHE) & chiral planar Hall effect (CPHE) \\
\hline
\end{tabular}

Under partial integration, one recognizes that the $A_{1}$ contributions combine into the Lifshitz invariant $(\boldsymbol{\nabla} \cdot \hat{\mathbf{n}}) n_{z}-(\hat{\mathbf{n}}$.

TABLE III. Classification of electric transport effects in a noncollinear magnet. For the symmetry group $C_{6 v}$, the conductivity tensor in the $x-y$ plane factors into the irreducible representations $\sigma=E_{1} \otimes E_{1}=A_{1}+A_{2}+E_{2}$. Each representation is generated from specific combinations of the components $\sigma_{\alpha \beta}$, which we refer to as channels. Within each channel, different kinds of physical effects can appear which are sensitive to different aspects of the underlying magnetization texture and which we roughly divide into those effects that are already present in a collinear ferromagnet and those that require a finite, first-order gradient $\partial_{i} n_{j}$. 
$\nabla) n_{z}$, which is also responsible for the DMI interaction under $C_{6 v}$ symmetry [39,40]. The two $E_{2}$ contributions are the same under partial integration as well. Combined, this leads to

$$
\begin{aligned}
\sigma_{A_{1}, \alpha \beta}^{\chi}(\hat{\mathbf{n}}) \partial_{\alpha} n_{\beta}= & \gamma_{\mathrm{CMC}}(\nabla \cdot \hat{\mathbf{n}}-\hat{\mathbf{n}} \cdot \nabla) n_{z}+O\left(n_{i}^{4}\right), \\
\sigma_{E_{2}, \alpha \beta}^{\chi}(\hat{\mathbf{n}}) \partial_{\alpha} n_{\beta}= & \gamma_{\mathrm{CPHE}} n_{z}\left(\partial_{x} n_{x}-\partial_{y} n_{y}, \partial_{x} n_{y}+\partial_{y} n_{x}\right) \\
& +O\left(n_{i}^{4}\right),
\end{aligned}
$$

describing the chiral magnetoconductivity (CMC) and the chiral planar Hall effect (CPHE).

The symmetrized tensor product $\operatorname{Sym}(\hat{\mathbf{n}} \otimes \hat{\mathbf{n}})$ carries the representation $\Gamma_{\operatorname{Sym}(\hat{\mathbf{n}} \otimes \hat{\mathbf{n}})}=2 A_{1}+E_{1}+E_{2}$. For the next order in $n_{i}$, one therefore finds $\Gamma_{\nabla \otimes \hat{\mathbf{n}}} \otimes \Gamma_{\operatorname{Sym}(\hat{\mathbf{n}} \otimes \hat{\mathbf{n}})}=4 A_{1}+4 A_{2}+$ $2 B_{1}+2 B_{2}+6 E_{1}+6 E_{2}$. Since this order is odd under time reversal, we are only interested in the four $A_{2}$ representations given by

$$
\begin{aligned}
A_{2} \otimes A_{1} \rightarrow & 2 A_{2}:(\nabla \cdot \hat{\mathbf{n}}) n_{\perp}^{2},(\nabla \cdot \hat{\mathbf{n}}) n_{\|}^{2}, \\
E_{1} \otimes E_{1} \rightarrow & A_{2}:(\hat{\mathbf{n}} \cdot \nabla) n_{\perp}^{2} / 2, \\
E_{2} \otimes E_{2} \rightarrow & A_{2}: n_{x} n_{y}\left(\partial_{x} n_{y}+\partial_{y} n_{x}\right) \\
& +\left(n_{x}^{2}-n_{y}^{2}\right)\left(\partial_{x} n_{x}-\partial_{y} n_{y}\right) / 2 .
\end{aligned}
$$

The last term may be recombined with other $A_{2}$ representations of this order such that it can be written as $(\hat{\mathbf{n}} \cdot \nabla) n_{\|}^{2} / 2$. And therefore

$$
\sigma_{A_{2}, \alpha \beta}^{\chi}(\hat{\mathbf{n}}) \partial_{\alpha} n_{\beta}=\gamma_{\mathrm{CHE}}(\nabla \cdot \hat{\mathbf{n}})\left(n_{\perp}^{2}-n_{\|}^{2}\right)+O\left(n_{i}^{5}\right)
$$

is the only coupling that describes the chiral Hall effect (CHE) in $C_{6 v}$ symmetric systems. In summary, we find that

$$
\begin{aligned}
\sigma_{A_{1}}[\hat{\mathbf{n}}]= & \left(\gamma_{\mathrm{LC}}+\gamma_{\mathrm{MC}}\right)+\gamma_{\mathrm{AMC}}\left\langle n_{\perp}^{2}-n_{\|}^{2}\right\rangle \\
& +\gamma_{\mathrm{CMC}}\left\langle(\nabla \cdot \hat{\mathbf{n}}-\hat{\mathbf{n}} \cdot \nabla) n_{z}\right\rangle+O\left(n_{i}^{4}\right) \\
\sigma_{A_{2}}[\hat{\mathbf{n}}]= & \gamma_{\mathrm{AHE}}\left\langle n_{z}\right\rangle+\gamma_{\mathrm{CHE}}\left\langle(\nabla \cdot \hat{\mathbf{n}})\left(n_{\perp}^{2}-n_{\|}^{2}\right)\right\rangle+O\left(n_{i}^{5}\right), \\
\sigma_{E_{2}}[\hat{\mathbf{n}}]= & \gamma_{\mathrm{PHE}}\left\langle\left[\left(n_{x}^{2}-n_{y}^{2}\right) / 2, n_{x} n_{y}\right]\right\rangle \\
& +\gamma_{\mathrm{CPHE}}\left\langle n_{z}\left(\partial_{x} n_{x}-\partial_{y} n_{y}, \partial_{x} n_{y}+\partial_{y} n_{x}\right)\right\rangle \\
& +O\left(n_{i}^{4}\right)
\end{aligned}
$$

where the factors $\gamma_{i}$ are temperature-dependent material constants that can be extracted from an underlying microscopic model. Inserting the spin-spiral defined by the combination of Eqs. (3) and (4), one arrives at

$$
\begin{aligned}
\sigma_{A_{1}}[\hat{\mathbf{n}}]= & \left(\gamma_{\mathrm{LC}}+\gamma_{\mathrm{MC}}\right)+\frac{1}{2} \gamma_{\mathrm{AMC}} \cos ^{2}(\alpha)[3 \cos (2 \alpha)-1] \\
& +\gamma_{\mathrm{CMC}} q \cos \beta \sin ^{3} \alpha+O\left(n_{i}^{4}\right) \\
\sigma_{A_{2}}[\hat{\mathbf{n}}]= & \gamma_{\mathrm{AHE}} \cos ^{2} \alpha+\frac{1}{2} \gamma_{\mathrm{CHE}} q \cos \beta \sin (\alpha) \sin ^{2}(2 \alpha) \\
& +O\left(n_{i}^{5}\right) \\
\sigma_{E_{2}}[\hat{\mathbf{n}}]= & -\frac{1}{8} \gamma_{\mathrm{PHE}} \sin ^{2}(\alpha)[3 \cos (2 \alpha)+1] \vartheta_{2} \\
& +\frac{1}{2} \gamma_{\mathrm{CPHE}} q \sin ^{3}(\alpha) \vartheta_{1}+O\left(n_{i}^{4}\right)
\end{aligned}
$$

where $\vartheta_{n}=\left(\cos \left(n \beta-2 \phi_{q}\right),-\sin \left(n \beta-2 \phi_{q}\right)\right)$.

\section{Kubo formalism}

Given specific electronic structure, we calculate the transverse and diagonal conductivity at zero temperature using the
Kubo formalism, which allows us to take into account the effect of disorder in the system on the conductivity tensor. To do so, we replace the retarded and advanced Green functions $G_{0}$ of the perfect crystal by the full Green function $G=\frac{1}{G_{0}^{-1}-\Sigma}$, where $\Sigma(E, \mathbf{k})$ is the self-energy representing the effect of disorder. Within this work, we are using a constant broadening model such that $\Sigma(E, \mathbf{k})=-i \Gamma \mathcal{I}$. With the constant broadening $\Gamma$ we obtain a Green function diagonal in the eigenspace of the Hamiltonian:

$$
G^{R / A}(E, \mathbf{k})_{m n}=\frac{\delta_{m n}}{E-\epsilon_{n \mathbf{k}} \pm i \Gamma},
$$

where $\epsilon_{n \mathbf{k}}$ are the single-electron eigenenergies. The antisymmetric part of the conductivity tensor, which can be expressed in terms of $G^{R / A}$ [41], splits into two contributions:

$$
\begin{aligned}
\sigma_{[\alpha \beta]}^{\mathrm{I}}= & -\frac{1}{2 \pi} \int \frac{d^{3} k}{(2 \pi)^{3}} \sum_{\substack{m n \\
m \neq n}} \operatorname{Im}\left\{v_{m n}^{\alpha}(\mathbf{k}) v_{n m}^{\beta}(\mathbf{k})\right\} \\
& \times \frac{\left(\epsilon_{m \mathbf{k}}-\epsilon_{n \mathbf{k}}\right) \Gamma}{\left[\left(E_{F}-\epsilon_{m \mathbf{k}}\right)^{2}+\Gamma^{2}\right]\left[\left(E_{F}-\epsilon_{n \mathbf{k}}\right)^{2}+\Gamma^{2}\right]}
\end{aligned}
$$

and

$$
\begin{aligned}
\sigma_{[\alpha \beta]}^{\mathrm{II}}= & -\frac{1}{\pi} \int \frac{d^{3} k}{(2 \pi)^{3}} \sum_{\substack{m \neq n \\
m \neq n}} \operatorname{Im}\left\{v_{m n}^{\alpha}(\mathbf{k}) v_{n m}^{\beta}(\mathbf{k})\right\} \\
& \times \frac{\Gamma}{\left(\epsilon_{m \mathbf{k}}-\epsilon_{n \mathbf{k}}\right)\left[\left(E_{F}-\epsilon_{m \mathbf{k}}\right)^{2}+\Gamma^{2}\right]} \\
& -\frac{1}{\left(\epsilon_{m \mathbf{k}}-\epsilon_{n \mathbf{k}}\right)^{2}} \operatorname{Im}\left\{\ln \left(\frac{E_{F}-\epsilon_{m \mathbf{k}}+i \Gamma}{E_{F}-\epsilon_{m \mathbf{k}}+i \Gamma}\right)\right\},
\end{aligned}
$$

where $\alpha$ and $\beta$ are the Cartesian indices, and $E_{F}$ is the Fermi energy. We refer to $\sigma_{\alpha \beta}^{\mathrm{I}}$ as the Fermi-surface term, since it only picks up contributions from the Fermi surface. The term $\sigma_{\alpha \beta}^{\mathrm{II}}$ collects terms from all occupied states up to the Fermi level and is therefore referred to as the Fermi-sea term. The symmetric part of the conductivity tensor is given by [42]

$$
\begin{aligned}
\sigma_{(\alpha \beta)}= & \frac{1}{\pi} \int \frac{d^{3} k}{(2 \pi)^{3}} \sum_{m n} \operatorname{Re}\left\{v_{m n}^{\alpha}(\mathbf{k}) v_{n m}^{\beta}(\mathbf{k})\right\} \\
& \times \frac{\Gamma^{2}}{\left[\left(E_{F}-\epsilon_{m \mathbf{k}}\right)^{2}+\Gamma^{2}\right]\left[\left(E_{F}-\epsilon_{n \mathbf{k}}\right)^{2}+\Gamma^{2}\right]} .
\end{aligned}
$$

In evaluating the Kubo expressions for the conductivity of spin-spiral states, we have considered systems treated in a supercell with up to 1600 atoms in the unit cell, using up to $2 \times 10^{5} k$-points for performing Brillouin zone integrations.

\section{CHIRAL TRANSPORT PROPERTIES OF SPIN-SPIRALS}

Below we compare the results of the gradient expansion performed up to linear order for the considered model to the explicit tight-binding calculations of the conductivity tensor by using the Kubo formalism. From explicit calculations of the conductivity for the system in a spin-spiral state $\hat{\mathbf{n}}_{\text {cone }}$ [defined in Eqs. (3) and (4)], we extract the contributions to the conductivity tensor which are even (nonchiral, $\sigma^{\text {nc }}$ ) and odd (chiral, $\sigma^{c}$ ) in spiral wave-vector $\mathbf{q}$ by performing the 
TABLE IV. The existence of chiral magnetotransport of spinspiral states. For each type of spiral state (Néel, Bloch, cone), the emergence of the corresponding effect [nonchiral (AHE) and chiral (CHE) anomalous Hall effect, nonchiral (MC) and chiral (CMC) magnetoconductivity, and nonchiral (PHE) and chiral (CPHE) planar Hall effect] is marked with the sign " $\checkmark$ " when it is allowed by symmetry arguments of the gradient expansion and confirmed by explicit Kubo transport calculations, while its absence is marked with an empty entry. Crystallographic directions [100], [0111], and [110] mark the direction of the $\mathbf{q}$-vector, which correspond to the value of $\phi_{q}$ of $0,-\pi / 2$, and $\pi / 3$, respectively.

\begin{tabular}{cccccccc}
\hline \hline \multicolumn{2}{c}{ Type } & AHE & CHE & MC & CMC & PHE & CPHE \\
\hline \multirow{6}{*}{ Néel } & {$[100]$} & & & $\checkmark$ & $\checkmark$ & & \\
& {$[0 \overline{1} 1]$} & & & $\checkmark$ & $\checkmark$ & & \\
\multirow{4}{*}{ Bloch } & {$[110]$} & & & $\checkmark$ & $\checkmark$ & $\checkmark$ & $\checkmark$ \\
& {$[100]$} & & & $\checkmark$ & & & $\checkmark$ \\
& {$[0 \overline{1} 1]$} & & & $\checkmark$ & & & $\checkmark$ \\
\multirow{6}{*}{ Cone } & {$[110]$} & & & $\checkmark$ & $\checkmark$ & $\checkmark$ & $\checkmark$ \\
& {$[100]$} & $\checkmark$ & $\checkmark$ & $\checkmark$ & $\checkmark$ & & \\
& {$[0 \overline{1} 1]$} & $\checkmark$ & $\checkmark$ & $\checkmark$ & $\checkmark$ & & \\
& {$[110]$} & $\checkmark$ & $\checkmark$ & $\checkmark$ & $\checkmark$ & $\checkmark$ & $\checkmark$ \\
\hline \hline
\end{tabular}

corresponding decomposition:

$$
\sigma_{\alpha \beta}^{c(\mathrm{nc})}=\frac{\sigma_{\alpha \beta}(\mathbf{q}) \mp \sigma_{\alpha \beta}(-\mathbf{q})}{2} .
$$

Using this definition, we can make a connection to the gradient expansion in the long-wavelength limit. Here, one finds the asymptotic relationships

$$
\begin{aligned}
& \sigma_{\alpha \beta}^{\mathrm{nc}} \sim\left\langle\sigma_{\alpha \beta}^{\mathrm{col}}(\hat{\mathbf{n}})\right\rangle \text { for } q \rightarrow 0, \\
& \sigma_{\alpha \beta}^{c} \sim\left\langle\sigma_{\alpha \beta \gamma \delta}^{\chi}(\hat{\mathbf{n}}) \partial_{\gamma} n_{\delta}\right\rangle \text { for } q \rightarrow 0 .
\end{aligned}
$$

We thus scrutinize the existence of chiral contributions to the magnetoconductivity, $\sigma_{(\alpha \alpha)}^{c}$ (q-chiral part of the diagonal components of the conductivity tensor $\sigma_{\alpha \alpha}$ ), the chiral planar Hall effect, $\sigma_{(\alpha \beta)}^{c}$ (q-chiral part of the $\alpha \leftrightarrow \beta$ symmetric off-diagonal components of the conductivity tensor $\sigma_{\alpha \beta}$ ), and the chiral Hall effect, $\sigma_{[\alpha \beta]}^{c}$ (q-chiral part of the $\alpha \leftrightarrow \beta$ antisymmetric off-diagonal components of the conductivity tensor $\left.\sigma_{\alpha \beta}\right)$. The predictions of the explicit calculations concerning the existence of MC and CMC, PHE and CPHE, and AHE and CHE are presented in Table IV. They are entirely consistent with the symmetry analysis of the gradient expansion also beyond the long-wavelength limit: whenever an empty instance is met in the table, the gradient expansion predicts a vanishing contribution for a given direction of the spin-spiral and its type, while explicit calculations provide negligible values of the conductivity. Below, we discuss in detail the emergence of chiral contributions to the MC, PHE, and AHE.

\section{A. Longitudinal chiral conductivity}

The discussion of chiral effects in the longitudinal conductivity falls into two categories: the isotropic contributions $\left(\sigma_{(x x)}+\sigma_{(y y)}\right) / 2$ from the totally symmetric irrep $A_{1}$, which we defined as CMC, and anisotropic contributions $\left(\sigma_{(x x)}-\right.$ $\left.\sigma_{(y y)}\right) / 2$ from irrep $E_{2}$, which we referred to as CLPHE. For the spiral, the two effects evaluate to

$$
\begin{gathered}
\gamma_{\mathrm{CMC}}\left\langle(\nabla \cdot \hat{\mathbf{n}}-\hat{\mathbf{n}} \cdot \nabla) n_{z}\right\rangle=\gamma_{\mathrm{CMC}} q \cos \beta \sin ^{3} \alpha, \\
\gamma_{\mathrm{CPHE}}\left\langle n_{z}\left(\partial_{x} n_{x}-\partial_{y} n_{y}\right)\right\rangle=\frac{1}{2} \gamma_{\mathrm{CPHE}} q \sin ^{3}(\alpha) \cos \left(\beta-2 \phi_{q}\right) .
\end{gathered}
$$

For a Bloch-type spiral, $\beta=\pi / 2$, the CMC is zero and the CLPHE terms only exist along specific directions: $\cos (\pi / 2-$ $\left.2 \phi_{q}\right)=\sin \left(2 \phi_{q}\right)$. From this argument it is clear that a Bloch spiral along the $x$-direction will not display any chiral longitudinal conductivity. Consequently, we take these longitudinal effects under closer inspection for a Néel-type spiral, $\beta=0$. Inspecting Eq. (40), we realize that

$$
\sigma_{(x x)}^{c}=\sigma_{A_{1}}+\sigma_{E_{2}}^{1}
$$

contains both isotropic and anisotropic components. However, both isotropic and anisotropic components follow the angular dependence dictated by symmetry,

$$
\sigma_{(x x)}^{c}(\alpha)=\left.\sin ^{3} \alpha \sigma_{(x x)}^{c}\right|_{\alpha=\pi / 2}
$$

in the long-wavelength limit, independent of the type of spiral.

The band structure of the model for the Néel spiral with $\pm \mathbf{q}$ along $x$ and the magnitude of $|\mathbf{q}|=0.31 a_{0}^{-1}$, presented in Fig. 2(a), shows a strong chiral asymmetry. Drastic modifications brought to the energetic positions of the states as well as their relative splittings give rise to a pronounced chiral contribution to the longitudinal conductivity. In Fig. 3(a) we show the CMC-signal given by $\sigma_{x x}^{c}$ as a function of Fermi energy $E_{f}$ and broadening $\Gamma$, taking a specific value of $|\mathbf{q}|=0.47 a_{0}^{-1}$ for a Néel spiral propagating along the $x$-axis. For comparison, we show the nonchiral MC in an inset. We observe the particle-hole symmetry of the model which is evident from the symmetry of $\sigma_{x x}^{c}$ with respect to Fermi energy $E_{f}$. This symmetry is fulfilled by all quantities displayed in Figs. 3(a)-3(f). Strong contributions to CMC, shown in Fig. 3(a), arise from certain specific energies (e.g., $\pm 1.3 \mathrm{eV}$ and broader humps around $\pm 2.0 \mathrm{eV}$ ), corresponding to the features in the electronic structure which are most affected by chirality. In contrast, nonchiral MC given by $\sigma_{x x}^{\text {nc }}$ is less sensitive to the Fermi energy. The magnitude of the computed CMC signal reaches as much as $30 \%$ of the nonchiral part of the conductivity. Clearly, the largest values of CMC are reached for the smallest values of $\Gamma$, and the rapid decay of $\sigma_{x x}^{c}$ with $\Gamma$ is evident. A careful analysis of the CMC scaling with $\Gamma$ for very small values of $\Gamma$ reveals an expected $1 / \Gamma$ behavior.

In Eq. (43) we uncovered a direct connection between the CMC for a Néel spiral and that of the cone-type spiral, valid in the limit of small spin-orbit coupling (SOC). This connection is intuitive in the sense that the Néel spiral is the limiting case of $\beta=0$ for the cone-type spiral, where the cone angle approaches $\alpha=\pi / 2$. We examine the prediction of Eq. (43) made from the relation between the Néel and the cone-type spiral in Fig. 4(a), comparing the scaling of the CMC-signal for the cone-type spiral, $\sigma_{x x \text {,cone }}^{c}$ (blue), to the function $\sin ^{3}(\alpha) \sigma_{x x \text {, Nél }}^{c}$ (red). For SOC strength of up to $\alpha_{R}=0.04 \mathrm{eV}$ we observe a perfect match where the cone-type spiral is equivalent to a Néel spiral (left side, $\alpha=0$ ) up to cone angles as large as $\pi / 4$. For even larger cone angles, 
(a)

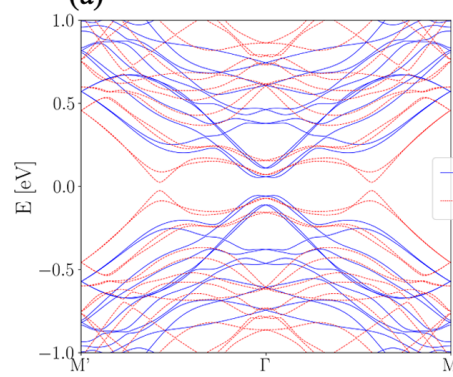

(e)

(b)

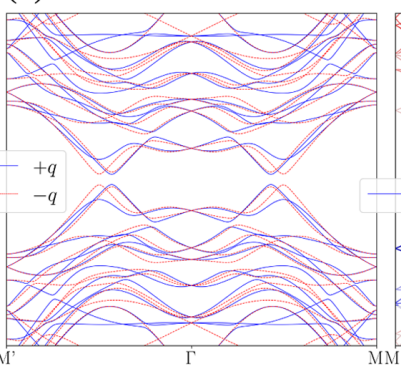

(f) (c)

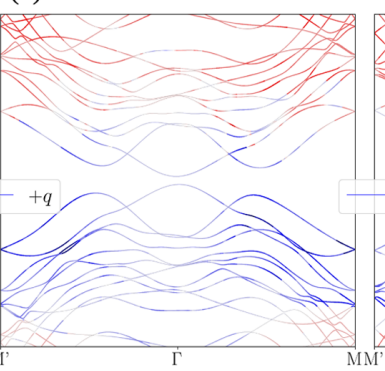

(g) (d)

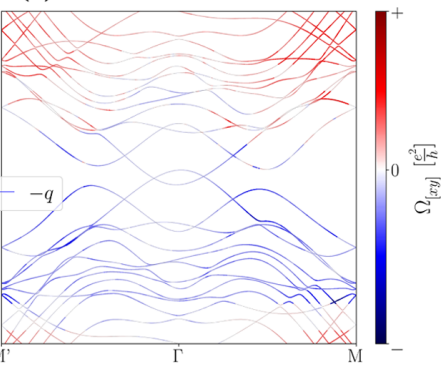

(h)

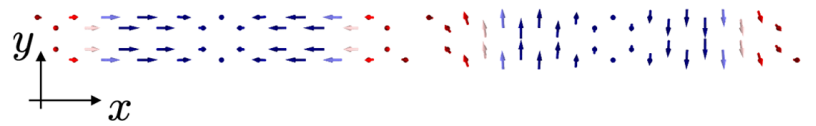

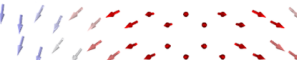

FIG. 2. Electronic structure of Néel, Bloch, and cone-type spirals. Band structures of Néel (a) and Bloch (b) spin-spirals in the energy interval $[-1,1] \mathrm{eV}$ for $\mathbf{q}= \pm 0.31 a_{0}^{-1} \cdot \hat{\boldsymbol{x}}$ (blue and red). (c),(d) Band structures of a cone-type spiral with the cone angle $\alpha=0.6 \mathrm{rad} \approx 34.38$ in the energy range of $[-1,1] \mathrm{eV}$ for opposite orientations of the wave vector $\mathbf{q}= \pm 0.31 a_{0}^{-1} \cdot \hat{\boldsymbol{x}}$, reflecting the changes in the electronic structure due to an opposite sense of chirality. The color of the bands in (c),(d) indicates the value of the band-resolved Berry curvature $\Omega_{x y}(\mathbf{k})$. (e)-(h) The real-space magnetic texture corresponding to band structures in the respective columns. For more details, see the main text.

the CMC signals deviate slightly (about 2-3\%) due to the influence of higher-order terms included in the numerical calculation, which are neglected in the gradient expansion. We can conclude here that the first nontrivial order in the gradient expansion reproduces the numerical calculations exceptionally well, when SOC is only of moderate strength.

The dependence of the CMC signal on the magnitude of $q$ is shown in Fig. 3(d) for a fixed value of $\Gamma$. Clearly, the spin-spiral pitch influences the magnitude of CMC in the most drastic way. The data reveal that CMC vanishes in the collinear ferromagnetic (FM) limit, $q=0$, and then quickly increases in magnitude over the whole energy range, as $q$ is increased, with the effect being particularly prominent at around $\pm 2 \mathrm{eV}$. Although the qualitative distinction between $\sim q$ and $\sim q^{2}$ contributions to the longitudinal conductivity of domain walls has been discussed in the past based on model arguments and material-specific calculations [25,32,43,44], the true chiral nature of the CMC manifests in the dependence of the overall signal on the sense of chirality of the spiral states observed here. Remarkably, the chiral magnetoconductivity may change sign several times for different values of $q$ at a fixed value of the Fermi energy. Such complex behavior finds its roots in a strong influence of the spin-spiral vector on the electronic structure of the system, with the corresponding shifts of the bands occurring over the range of eVs. CMC, therefore, directly stems from the presence of strong spin-orbit coupling, tying the rearrangements of bands to the sense of chirality as it is visible in Fig. 2. The extreme sensitivity of $\mathrm{CMC}$ to wave vector $q$ suggests that for spin-textures hosted in materials with strong spin-orbit interaction, the measured longitudinal conductivity can fluctuate dramatically even upon small variations in spin distribution, brought about, for example, by an external magnetic field. Naively, such changes in measured magnetotransport can be erroneously interpreted as arising from qualitative modifications in texture properties.

\section{B. Chiral planar Hall effect}

Traditionally, the planar Hall effect is understood as the $\sigma_{(x y)}$ entry of the conductivity tensor, which we find in the second component of irrep $E_{2}, \sigma_{(x y)}=\sigma_{E_{2,2}}[\hat{\mathbf{n}}]$. For the case of $C_{6 v}$ symmetry and the cone spiral as defined before, one finds the chiral contribution as a sum of two terms,

$$
\begin{aligned}
\sigma_{E_{2,2}}[\hat{\mathbf{n}}]= & \frac{1}{8} \gamma_{\mathrm{PHE}} \sin ^{2}(\alpha)[3 \cos (2 \alpha)+1] \sin \left(2 \beta-2 \phi_{q}\right) \\
& -\frac{1}{2} \gamma_{\mathrm{CPHE}} q \sin ^{3}(\alpha) \sin \left(\beta-2 \phi_{q}\right) .
\end{aligned}
$$

The nonchiral contribution vanishes exactly. For tilted conical Néel spirals, $\beta=0$, the angular dependence is $\sin \left(2 \phi_{q}\right)$ for both terms. As a direct consequence, the tensor component describing the CPHE for Néel spirals is only nonzero for $\phi_{q} \neq 0$, i.e., when the wave vector $\mathbf{q}$ is not parallel to the $x$-axis. In contrast, the term in $\sigma_{E_{2,2}}[\hat{\mathbf{n}}]$ proportional to $\gamma_{\mathrm{CPHE}}$ survives for the corresponding spirals of Bloch-type along the $x$-axis, $\beta=\pi / 2$ and $\phi_{q}=0$. This can be understood by realizing that for $\beta=\pi / 2$, the term linear in $\gamma_{\mathrm{CPHE}}$ is proportional to $\cos \left(2 \phi_{q}\right)$, while the term linear in $\gamma_{\mathrm{PHE}}$ is proportional to $\sin \left(2 \phi_{q}\right)$. This effective phase shift of $\pi / 2$ results in a nonzero contribution to the symmetric transverse conductivity $\sigma_{(x y)}$. Therefore, we investigate $\sigma_{(x y)}$ for the Bloch spiral along the $x$-axis, which has anisotropic contributions from irrep $E_{2}$ proportional to $\gamma_{\mathrm{CPHE}}$ only. This is in contrast to the case of $\sigma_{x x}$ calculated for Néel spirals along $x$, where both anisotropic and isotropic components from irreps $A_{1}$ and $E_{2}$ contribute.

The band structure of the model for a Bloch spiral with $\pm \mathbf{q}$ along $x$ and the magnitude of $|\mathbf{q}|=0.31 a_{0}^{-1}$, presented in Fig. 2(b), exhibits certain chiral asymmetry of the bands. In comparison to the band structure of the Néel spiral shown in Fig. 2(a), the rearrangement of bands is not as drastic for the Bloch spiral. Nonetheless, the chiral PHE is very prominent, as can be observed for a Bloch spiral propagating along the $x$-axis with $|\mathbf{q}|=0.47 a_{0}^{-1}$ in Fig. $3(\mathrm{~b})$. Similar to the CMCsignal shown in Fig. 3(a), most pronounced features in the CPHE originate from the energy regions around $\pm 1.3 \mathrm{eV}$ and around $\pm 2.0 \mathrm{eV}$. While these features are generally broader in energy and similar in magnitude to $\mathrm{CMC}$, strong fingerprints of CPHE appear also around $0 \mathrm{eV}$. In comparison to CMC, chiral PHE is not as rapidly decreasing with increasing $\Gamma$, although the overall trend remains. 


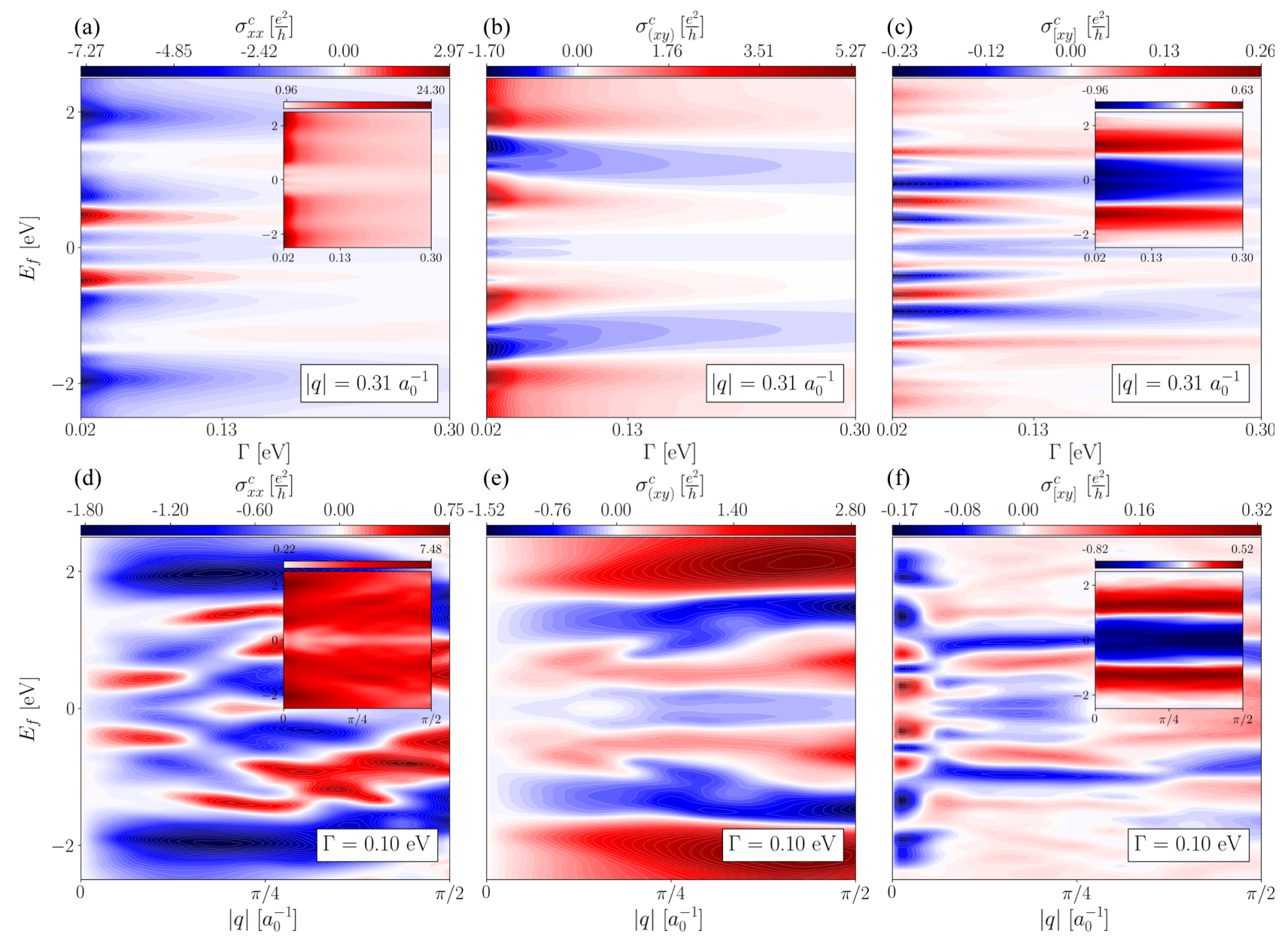

FIG. 3. Chiral magnetoconductivity (CMC), chiral planar Hall effect (CPHE), and chiral Hall effect (CHE). (a,d) Chiral magnetoconductivity (CMC), $\sigma_{x x}^{c}$, calculated for a Néel-type spiral with $\mathbf{q}= \pm 0.47 a_{0}^{-1} \cdot \hat{\boldsymbol{x}}$ as a function of Fermi energy $E_{f}$ and broadening $\Gamma$, (a), and as a function of $E_{f}$ and $q$ (along $\hat{\boldsymbol{x}}$ ) at $\Gamma$ of $100 \mathrm{meV}$, (d). The insets depict the corresponding behavior of the nonchiral part of the conductivity tensor element, $\sigma_{x x}^{\text {nc }}$. (b),(e) Chiral planar Hall effect (CPHE), $\sigma_{(x y)}^{c}$, calculated for a Bloch spiral with $\mathbf{q}= \pm 0.47 a_{0}^{-1} \cdot \hat{\boldsymbol{x}}$ as a function of $E_{f}$ and $\Gamma$, (b), and as a function of $E_{f}$ and $q$ (along $\hat{\boldsymbol{x}}$ ) at $\Gamma$ of $100 \mathrm{meV}$, (e). The corresponding nonchiral part of the conductivity tensor element, $\sigma_{(x x)}^{\text {nc }}$, vanishes in this case; see Table IV. (c),(f) Chiral Hall effect (CHE), $\sigma_{[x y]}^{c}$, calculated for a cone-type spiral with $\mathbf{q}= \pm 0.47 a_{0}^{-1} \cdot \hat{\boldsymbol{x}}$ and a cone angle of $\alpha=0.6 \mathrm{rad} \approx 34.38$ as a function of $E_{f}$ and $\Gamma$, (c), and as a function of $E_{f}$ and $q$ (along $\hat{x}$ ) at $\Gamma$ of $100 \mathrm{meV}$, (f). The insets depict the corresponding behavior of the nonchiral part of the conductivity tensor element, $\sigma_{[x y]}^{\mathrm{nc}}$.

Moving on to Fig. 3(d), we inspect CPHE as a function of $q$ for a fixed value of $\Gamma$. We observe that the CPHE signal vanishes for the FM limit and then increases in magnitude, but not as rapidly as the CMC signal does. Moreover, at fixed $E_{f}$, sign changes with $q$ occur only at very specific energies from the range around $\pm 1 \mathrm{eV}$ for the CPHE, and not over wide ranges of energies as is the case for CMC. This is a consequence of an overall smoother distribution of CPHE as a function of $E_{f}$ and $q$. We expect that CMC and CPHE are clearly distinguishable experimentally in materials displaying spiral textures, since they display opposite signs over a wide range of spiral pitch $q$. One of the most interesting features of the planar Hall effect for Bloch-type spirals propagating along the $x$-axis is suppressed nonchiral PHE, which is given by $\sigma_{(x y)}^{\text {nc }}$. This numerical result is in perfect agreement with the predictions from the gradient expansion; see Table IV. The nonchiral PHE vanishes completely, while we observe a planar Hall effect, comparable in magnitude to AMC, which is purely chiral in nature.

\section{Chiral Hall effect}

We finally discuss chiral corrections to the AHE exhibited by spin-spiral states. The AHE is described by the antisymmetric conductivity $\sigma_{[x y]}$, where we find the chiral term

$$
\gamma_{\mathrm{CHE}}\left\langle(\nabla \cdot \hat{\mathbf{n}})\left(n_{\perp}^{2}-n_{\|}^{2}\right)\right\rangle=\frac{1}{2} \gamma_{\mathrm{CHE}} q \cos \beta \sin (\alpha) \sin ^{2}(2 \alpha)
$$

for the cone spiral as introduced before. Correspondingly, this effect is referred to as the chiral Hall effect (CHE). It is zero for $\alpha=0^{\circ}$ and $90^{\circ}$, and it attains a maximum value at $\alpha \approx$ 50.77. We inspect the CHE-signal for the cone-type spiral at the intermediate value $\alpha=0.6 \mathrm{rad} \approx 34.38^{\circ}$. 
In Fig. 3(c) we present the calculated CHE-signal, as given by $\sigma_{[x y]}^{c}$, for the cone-type spiral propagating along the $x$-axis with $|\mathbf{q}|=0.47 a_{0}^{-1}$ and a cone angle $\alpha=0.6 \mathrm{rad}$ as a function of Fermi energy $E_{f}$ and broadening $\Gamma$. The overall observed structure of the CHE as a function of the Fermi energy is much richer than that exhibited by $\mathrm{CMC}$ and $\mathrm{CPHE}$, as well as the nonchiral part of the AHE, $\sigma_{[x y]}^{\text {nc }}$ (shown in the inset). Consequently, the CHE is characterized by a stronger sensitivity to the finer details of the electronic structure mediated by the sense of chirality. In comparison to CMC and CPHE, the most remarkable feature of both chiral and nonchiral parts of the AHE is a qualitatively different behavior with $\Gamma$ : in contrast to the data shown in Figs. 3(a) and 3(b) for CMC and CPHE, the CHE-signal does not scale as dramatically with the broadening. In particular, the most pronounced features of the CHE located in the energy region $[-1 \mathrm{eV}, 1 \mathrm{eV}]$ are the least sensitive to $\Gamma$ variation. This behavior can be explained by the robustness of intrinsic contributions to the $\mathrm{CHE}$. In fact, with decreasing disorder the values of the CHE converge to the clean limit values, which are given by the integrated values of Berry curvature $\Omega_{x y}$ antisymmetrized with respect to $\mathbf{q}$ [20]. The rise of nonvanishing intrinsic chiral AHE becomes apparent from looking at Figs. 2(c) and 2(d), where the strongly chirality-dependent electronic structure and Berry curvature of the states is visible. On the other hand, by comparing the chiral and nonchiral parts of the AHE, we observe that the disorder corrections to the intrinsic conductivity are much more important for the CHE than for the conventional AHE: the convergence to the clean-limit values is quite slow, and the saturation does not occur up to broadening $\Gamma$ on the scale of $0.01 \mathrm{meV}$. This points to possibly very prominent extrinsic contributions to the chiral Hall effect in realistic spin textures.

In Fig. 3(f) we analyze the behavior of the CHE signal with the magnitude of the wave vector $q$. What can be concluded from comparing all the effects among each other is that while the magnitude of the AHE and CHE is expectedly significantly smaller than that of the CMC and CPHE (at the same value of $\Gamma$ ), the magnitudes of the chiral and nonchiral AHE are comparable. Among the latter two, the $\mathrm{CHE}$ is much more sensitive to $q$, which is especially visible for smaller values of $q$, where the structure of the chiral signal is especially nontrivial with respect to the Fermi energy. Here, small deviations from the zero- $q$ limit may result in sign changes in $\mathrm{CHE}$, which is relevant for magnetotransport detection of textures (see the discussion of CMC). By inspecting the $q=0$ limit more closely in Fig. 4(b), we find that the data correctly reproduce the ferromagnetic limit, where the $\mathrm{CHE}$ is vanishing. The data presented in Fig. 4(c) also illustrate the linear scaling behavior of the CHE signal $\sigma_{[x y]}^{a}$ with increasing $q$ away from the ferromagnetic limit. This stands in agreement with the gradient expansion prediction, and it confirms that higher-order beyond-linear corrections to the chirality-sensitive signal are negligible in the limit of vanishing $q$.

The limit on the validity of the gradient expansion technique is apparent from the calculations: deviations from linear-in- $q$ behavior are visible above $q \approx 0.03 a_{0}^{-1}$ - a value that depends significantly on the parameters of the model. Importantly, in this range of the $q$-vectors, we observe that the nonchiral corrections to the $q=0$ values of the AHE
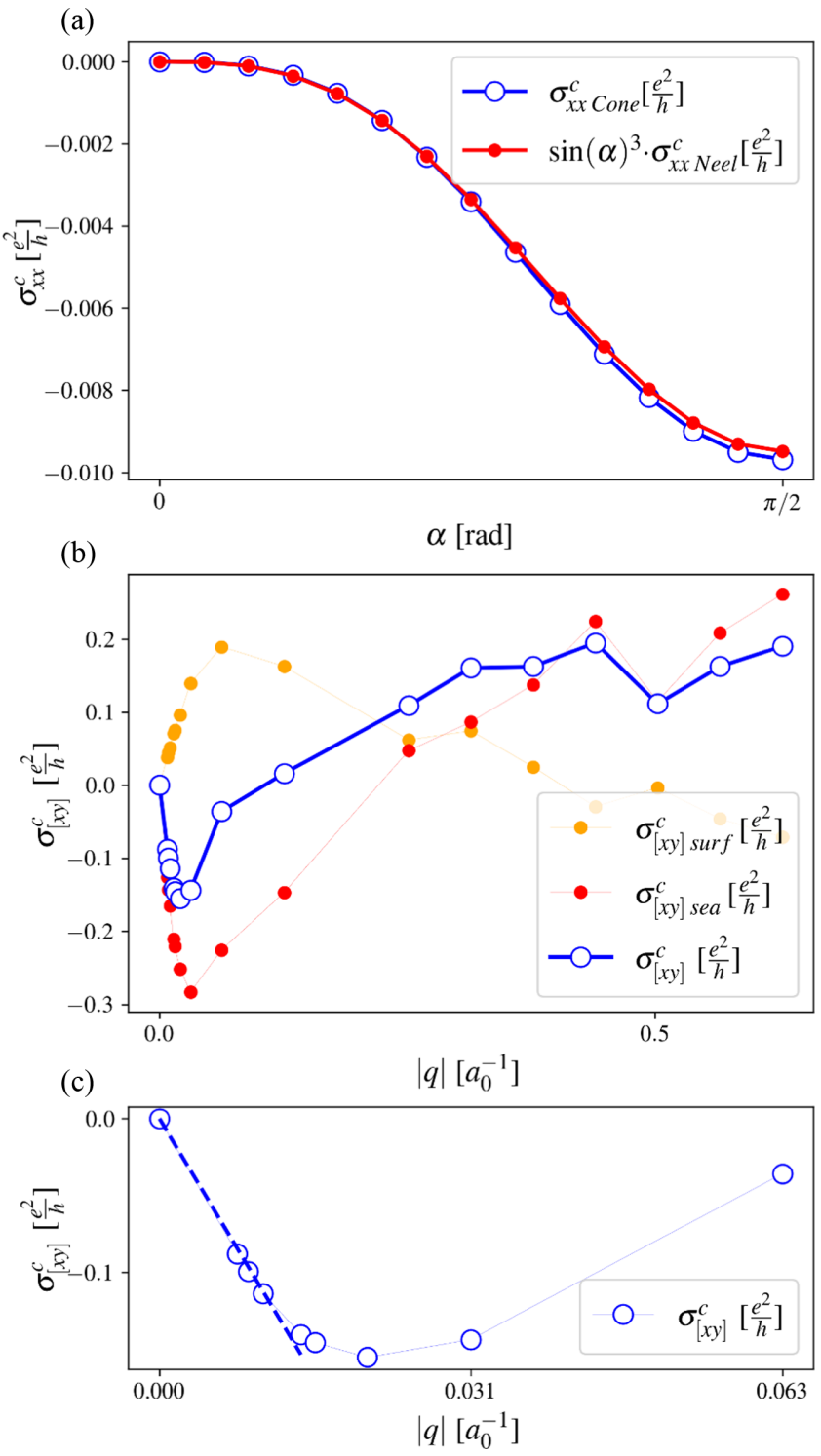

FIG. 4. Scaling with the cone angle and spin-spiral pitch. (a) Chiral magnetoconductivity (CMC) for a cone-type spiral (blue) calculated for cone angles in the range $\alpha \in[0, \pi / 2]$ and CMC for a Néel-type spiral (red) calculated for cone angle $\alpha=0$ and extrapolated to larger cone angles by multiplying with $\sin (\alpha)^{3}$; see (43). The relationship between CMC for cone-type and Néel spirals obtained from the gradient expansion is reproduced with explicit tight-binding calculations. The calculation was done for spin-orbit coupling $\alpha_{R}=$ $0.04 \mathrm{eV}$ at a spiral pitch $q=0.031 a_{0}^{-1}$. (b) Ferromagnetic limit of the chiral Hall effect (CHE) for a cone-type spiral. Surface term (orange), sea term (red), and the sum of the two (blue) yielding the CHE for nonzero broadening $\Gamma$. (c) The CHE signal near the FM limit of $q=0$. The linear slope $\gamma \approx-10.752 e^{2} a_{0} / h$ in the limit of $q \rightarrow 0$, participating in a gradient expansion prediction (50), can be estimated from calculations. For all plots, the data were calculated for the values of $E_{f}=-0.625 \mathrm{eV}$ and $\Gamma=0.2 \mathrm{eV}$. A value of $\alpha_{R}=0.4 \mathrm{eV}$ was used in (b) and (c).

are significantly smaller in magnitude than the chiral signal. This has consequences for our estimates of the qualitative importance of the linear versus higher order in the magnetization gradient contributions to the transport properties of the large-scale textures, as discussed below. 


\section{IMPACT ON TRANSPORT PROPERTIES OF SKYRMIONS}

While super cell calculations for one-dimensional textures are already computationally demanding, treating twodimensional textures such as a lattice of skyrmions requires even more resources. However, one can deduce certain transport properties in general slowly varying noncollinear textures from the properties of the spiral phase. For example, we consider a skyrmion texture defined by $\theta(r)=\pi(1-r / w)$, and $\quad \hat{\mathbf{n}}_{\mathrm{sk}}=(\sin (\theta) \cos (\varphi+\eta), \sin \theta \sin (\varphi+\eta), \cos (\theta))^{T}$, where $(r, \varphi)$ are polar coordinates in the plane. We imagine that the skyrmion $\hat{\mathbf{n}}_{\mathrm{sk}}$ is embedded in a ferromagnetic host, $\hat{\mathbf{n}}_{\mathrm{fm}}=\mathbf{e}_{z}$, and we integrate the real-space averages in Eqs. (27)-(29) within the volume of the skyrmion $\mathcal{V}=\pi w^{2}$. The helicity $\eta$ interpolates between the Néel skyrmion for $\eta=0$ and the Bloch skyrmion with $\eta=\pi / 2$. We can then estimate the change in conductivity which is induced by the skyrmion:

$$
\begin{aligned}
& \sigma_{A_{1}}^{\mathrm{sk}}-\sigma_{A_{1}}^{\mathrm{fm}}=-\frac{\pi}{w} \gamma_{\mathrm{CMC}} \cos \eta, \\
& \sigma_{A_{2}}^{\mathrm{sk}}-\sigma_{A_{2}}^{\mathrm{fm}}=\left(\frac{4}{\pi^{2}}-1\right) \gamma_{\mathrm{AHE}}-\frac{32}{9 \pi w} \gamma_{\mathrm{CHE}} \cos \eta, \\
& \sigma_{E_{2}}^{\mathrm{sk}}-\sigma_{E_{2}}^{\mathrm{fm}}=0 .
\end{aligned}
$$

The explicit helicity dependence is one of the hallmarks of "chiral" transport effects [19]. In contrast to the topological Hall effect, the CHE is further characterized by its $1 / w$ scaling dependence in the long-wavelength limit. In the case of the THE, a $1 / w^{2}$-scaling would be expected, originating from the emergent magnetic field $B_{\text {eff }} \sim \hat{\mathbf{n}} \cdot\left(\partial_{x} \hat{\mathbf{n}} \times \partial_{y} \hat{\mathbf{n}}\right)$ and which, for large $w$, will eventually be the subdominant contribution of the two different effects. Concerning practical computations, the coefficient $\gamma_{\text {AHE }}$ can be simply extracted from a calculation of a collinear state, while the material parameters $\gamma_{\mathrm{CMC}}$ and $\gamma_{\mathrm{CHE}}$ could be estimated from the spiral calculations as presented before via

$$
\begin{aligned}
\gamma_{\mathrm{CMC}} & \approx \lim _{q \rightarrow 0} \partial_{q} \frac{\sigma_{A_{1}}[\hat{\mathbf{n}}]}{\cos \beta \sin ^{3} \alpha}, \\
\gamma_{\mathrm{CHE}} & \approx \lim _{q \rightarrow 0} \partial_{q} \frac{\sigma_{A_{2}}[\hat{\mathbf{n}}]}{\cos \beta \sin \alpha \sin ^{2}(2 \alpha) / 2} .
\end{aligned}
$$

The relation is approximate since strong spin-orbit coupling would lead to higher-order corrections in the $\alpha$ and $\beta$ dependence which could not be factored out. Irrespective of this, the large effective values of $\gamma$ correspond to the observation that $\mathrm{CHE}$ reaches values comparable in magnitude to the collinear AHE already for small values of pitch $q$. This speaks to the fact that the chiral transport signal should be the dominant source of texture-driven conductivity for wide regimes of texture profiles.

\section{CONCLUSIONS}

In this work, we have pursued the idea that homogeneous spin-spiral states can exhibit macroscopic transport properties that are sensitive to their sense of winding, or chirality. Inspired by previous effective analysis, we referred to an explicit two-dimensional electronic model and rigorously demonstrated the emergence of chiral magnetoconductivity, the chiral planar Hall effect, and the chiral Hall effect. Based on Kubo and gradient expansion techniques, we showed that the corresponding chiral signal can be very prominent, depending on the type of the spiral state. It also follows from our analysis that in addition to the high sensitivity of the effects to the electronic structure, the lengthscale of the one-dimensional spin texture has a profound effect on the magnitude and sign of the chiral signal. This can prove extremely useful in dealing with aspects such as transport signatures of texture dynamics or magnetic phase transitions. Besides bringing fundamental novel insights into the interplay between spin chirality and magnetotransport, we show how an ability to predict the underlying properties of simpler spiral states paves the way to understanding and educated engineering of transport fingerprints for more complex textures such as magnetic skyrmions.

\section{ACKNOWLEDGMENTS}

We acknowledge funding under SPP 2137 "Skyrmionics" of the Deutsche Forschungsgemeinschaft (DFG, German Research Foundation). We also gratefully acknowledge the Jülich Supercomputing Centre and RWTH Aachen University for providing computational resources under Projects No. jiff40 and No. jpgi11. The work was also supported by the DFG-TRR 173-268565370 (Project A11), TRR 288422213477 (Project B06), and Project MO 1731/10-1 of the DFG. J.K. acknowledges funding under HGF-RSF Joint Research Group "TOPOMANN", Grant No. DB001827. The authors express their gratitude to Prof. Stefan Blügel and Dr. Juba Bouaziz for fruitful discussion.
[1] P. Bruno, V. K. Dugaev, and M. Taillefumier, Topological Hall Effect and Berry Phase in Magnetic Nanostructures, Phys. Rev. Lett. 93, 096806 (2004).

[2] G. Tatara and H. Kawamura, Chirality-driven anomalous hall effect in weak coupling regime, J. Phys. Soc. Jpn. 71, 2613 (2002).

[3] M. Onoda, G. Tatara, and N. Nagaosa, Anomalous hall effect and skyrmion number in real and momentum spaces, J. Phys. Soc. Jpn. 73, 2624 (2004).

[4] K. Nakazawa, M. Bibes, and H. Kohno, Topological hall effect from strong to weak coupling, J. Phys. Soc. Jpn. 87, 033705 (2018).
[5] C. Franz, F. Freimuth, A. Bauer, R. Ritz, C. Schnarr, C. Duvinage, T. Adams, S. Blügel, A. Rosch, Y. Mokrousov, and C. Pfleiderer, Real-Space and Reciprocal-Space Berry Phases in the Hall Effect of $\mathrm{Mn}_{1-x} \mathrm{Fe}_{x} \mathrm{Si}$, Phys. Rev. Lett. 112, 186601 (2014).

[6] C. S. Spencer, J. Gayles, N. A. Porter, S. Sugimoto, Z. Aslam, C. J. Kinane, T. R. Charlton, F. Freimuth, S. Chadov, S. Langridge, J. Sinova, C. Felser, S. Blügel, Y. Mokrousov, and C. H. Marrows, Helical magnetic structure and the anomalous and topological Hall effects in epitaxial b20 $\mathrm{Fe}_{1-y} \mathrm{Co}_{y} \mathrm{Ge}$ films, Phys. Rev. B 97, 214406 (2018). 
[7] A. Neubauer, C. Pfleiderer, B. Binz, A. Rosch, R. Ritz, P. G. Niklowitz, and P. Böni, Topological Hall Effect in the $A$ Phase of MnSi, Phys. Rev. Lett. 102, 186602 (2009).

[8] R. Ritz, M. Halder, C. Franz, A. Bauer, M. Wagner, R. Bamler, A. Rosch, and C. Pfleiderer, Giant generic topological Hall resistivity of mnsi under pressure, Phys. Rev. B 87, 134424 (2013).

[9] M. Hirschberger, T. Nakajima, M. Kriener, T. Kurumaji, L. Spitz, S. Gao, A. Kikkawa, Y. Yamasaki, H. Sagayama, H. Nakao, S. Ohira-Kawamura, Y. Taguchi, T.-h. Arima, and Y. Tokura, High-field depinned phase and planar Hall effect in the skyrmion host $\mathrm{Gd}_{2} \mathrm{PdSi}_{3}$, Phys. Rev. B 101, 220401(R) (2020).

[10] P. Vir, J. Gayles, A. S. Sukhanov, N. Kumar, F. Damay, Y. Sun, J. Kübler, C. Shekhar, and C. Felser, Anisotropic topological Hall effect with real and momentum space Berry curvature in the antiskyrmion-hosting Heusler compound $\mathrm{Mn}_{1.4} \mathrm{PtSn}$, Phys. Rev. B 99, 140406(R) (2019).

[11] V. Kumar, N. Kumar, M. Reehuis, J. Gayles, A. S. Sukhanov, A. Hoser, F. M. C. Damay, C. Shekhar, P. Adler, and C. Felser, Detection of antiskyrmions by topological Hall effect in Heusler compounds, Phys. Rev. B 101, 014424 (2020).

[12] P. Swekis, J. Gayles, D. Kriegner, G. H. Fecher, Y. Sun, S. T. B. Goennenwein, C. Felser, and A. Markou, Role of magnetic exchange interactions in chiral-type hall effects of epitaxial mnxptsn films, ACS Appl. Electron. Mater. 3, 1323 (2021).

[13] B. Göbel, I. Mertig, and O. A. Tretiakov, Beyond skyrmions: Review and perspectives of alternative magnetic quasiparticles, Phys. Rep. 895, 1 (2021.

[14] T. Yamaguchi and A. Yamakage, Theory of magnetic-textureinduced anomalous hall effect on the surface of topological insulators, J. Phys. Soc. Jpn. 90, 063703 (2021).

[15] M. Hirschberger, Y. Nomura, H. Mitamura, A. Miyake, T. Koretsune, Y. Kaneko, L. Spitz, Y. Taguchi, A. Matsuo, K. Kindo, R. Arita, M. Tokunaga, and Y. Tokura, Geometrical Hall effect and momentum-space Berry curvature from spin-reversed band pairs, Phys. Rev. B 103, L041111 (2021).

[16] F. R. Lux, F. Freimuth, S. Blügel, and Y. Mokrousov, Engineering chiral and topological orbital magnetism of domain walls and skyrmions, Commun. Phys. 1, 60 (2018).

[17] J. Bouaziz, H. Ishida, S. Lounis, and S. Blügel, Transverse Transport in Two-Dimensional Relativistic Systems with Nontrivial Spin Textures, Phys. Rev. Lett. 126, 147203 (2021).

[18] F. R. Lux, F. Freimuth, S. Blügel, and Y. Mokrousov, Chiral Hall Effect in Noncollinear Magnets from a Cyclic Cohomology Approach, Phys. Rev. Lett. 124, 096602 (2020).

[19] M. Redies, F. R. Lux, J.-P. Hanke, P. M. Buhl, S. Blügel, and Y. Mokrousov, Mixed topology ring states for Hall effect and orbital magnetism in skyrmions of Weyl semimetals, Phys. Rev. B 102, 184407 (2020).

[20] J. Kipp, K. Samanta, F. R. Lux, M. Merte, J. P. Hanke, M. Redies, F. Freimuth, S. Blügel, M. Ležaić, and Y. Mokrousov, The chiral hall effect in canted ferromagnets and antiferromagnets, Commun. Phys. 4, 99 (2021).

[21] S. A. Meynell, M. N. Wilson, J. C. Loudon, A. Spitzig, F. N. Rybakov, M. B. Johnson, and T. L. Monchesky, Hall effect and transmission electron microscopy of epitaxial mnsi thin films, Phys. Rev. B 90, 224419 (2014).
[22] M. Bode, M. Heide, K. von Bergmann, P. Ferriani, S. Heinze, G. Bihlmayer, A. Kubetzka, O. Pietzsch, S. Blügel, and R. Wiesendanger, Chiral magnetic order at surfaces driven by inversion asymmetry, Nature (London) 447, 190 (2007).

[23] M. Menzel, Y. Mokrousov, R. Wieser, J. E. Bickel, E. Vedmedenko, S. Blügel, S. Heinze, K. von Bergmann, A. Kubetzka, and R. Wiesendanger, Information Transfer by Vector Spin Chirality in Finite Magnetic Chains, Phys. Rev. Lett. 108, 197204 (2012).

[24] J. Spethmann, S. Meyer, K. von Bergmann, R. Wiesendanger, S. Heinze, and A. Kubetzka, Discovery of Magnetic Single- and Triple-q States in Mn/Re(0001), Phys. Rev. Lett. 124, 227203 (2020).

[25] K. M. Seemann, F. Garcia-Sanchez, F. Kronast, J. Miguel, A. Kákay, C. M. Schneider, R. Hertel, F. Freimuth, Y. Mokrousov, and S. Blügel, Disentangling the Physical Contributions to the Electrical Resistance in Magnetic Domain Walls: A Multiscale Study, Phys. Rev. Lett. 108, 077201 (2012).

[26] S. Heinze, K. von Bergmann, M. Menzel, J. Brede, A. Kubetzka, R. Wiesendanger, G. Bihlmayer, and S. Blügel, Spontaneous atomic-scale magnetic skyrmion lattice in two dimensions, Nat. Phys. 7, 713 (2011).

[27] F. Freimuth, S. Blügel, and Y. Mokrousov, Anisotropy and temperature-dependence of spin-orbit torques: Role of magnons, Phys. Rev. B 104, 094434 (2021).

[28] L.-c. Zhang, D. Go, J.-P. Hanke, P. M. Buhl, S. Grytsiuk, F. R. Lux, S. Blügel, and Y. Mokrousov, Imprinting and driving electronic orbital magnetism using magnons, Commun. Phys. 3, 227 (2020).

[29] F. R. Lux, P. Prass, S. Blügel, and Y. Mokrousov, Effective seiberg-witten gauge theory of noncollinear magnetism, arXiv:2005.12629.

[30] L. M. Sandratskii, Energy band structure calculations for crystals with spiral magnetic structure, Phys. Status Solidi B 136, 167 (1986)

[31] L. M. Sandratskii, Symmetry analysis of electronic states for crystals with spiral magnetic order. i. general properties, J. Phys.: Condens. Matter 3, 8565 (1991).

[32] Z. Yuan, Y. Liu, A. A. Starikov, P. J. Kelly, and A. Brataas, Spin-Orbit-Coupling-Induced Domain-Wall Resistance in Diffusive Ferromagnets, Phys. Rev. Lett. 109, 267201 (2012).

[33] R. R. Birss, Symmetry and Magnetism (North-Holland, Amsterdam, 1964)

[34] A. Aqeel, M. Azhar, N. Vlietstra, A. Pozzi, J. Sahliger, H. Huebl, T. T. M. Palstra, C. H. Back, and M. Mostovoy, Allelectrical detection of skyrmion lattice state and chiral surface twists, Phys. Rev. B 103, L100410 (2021).

[35] M. S. Dresselhaus, G. Dresselhaus, and A. Jorio, Group Theory: Application to the Physics of Condensed Matter (Springer, Berlin, Heidelberg, 2007).

[36] M. Hamermesh, Group Theory and Its Application to Physical Problems, Dover Books on Physics (Dover Publications, New York, 2012).

[37] F. E. Neumann, Vorlesungen über die Theorie der Elasticität der festen Körper und des Lichtäthers: Gehalten an der Universität Königsberg (BG Teubner, Leipzig, 1885), Vol. 4.

[38] A. S. Nowick, Crystal Properties Via Group Theory (Cambridge University Press, Cambridge, 2005). 
[39] I. E. Dzyaloshinskii, Theory of helicoidal structures in antiferromagnets. i. nonmetals, Sov. Phys. JETP 19, 960 (1964).

[40] A. N. Bogdanov and D. Yablonskii, Thermodynamically stable "vortices" in magnetically ordered crystals. the mixed state of magnets, Zh. Eksp. Teor. Fiz. 95, 178 (1989).

[41] P. Czaja, F. Freimuth, J. Weischenberg, S. Blügel, and Y. Mokrousov, Anomalous Hall effect in ferromagnets with Gaussian disorder, Phys. Rev. B 89, 014411 (2014).

[42] P. Czaja, Anomalous Hall effect and orbital magnetism in disordered ferromagnets from Ab Initio, Master's thesis, Department of Physics, RWTH Aachen University, Germany, 2013.
[43] B. Y. Yavorsky, I. Mertig, A. Y. Perlov, A. N. Yaresko, and V. N. Antonov, Giant magnetoresistance due to a domain wall in Fe: Ab initio study, Phys. Rev. B 66, 174422 (2002).

[44] J. B. A. N. van Hoof, K. M. Schep, A. Brataas, G. E. W. Bauer, and P. J. Kelly, Ballistic electron transport through magnetic domain walls, Phys. Rev. B 59, 138 (1999).

Correction: The previously published Figure 3 contained insufficient data for the range of $\Gamma$ in the upper panels and the range of $q$ in the lower panels and has been replaced. 